ISSN 1112-9867

http://www.jfas.info

\title{
FLOW SIMULATION AND INVESTIGATING THE EFFECTS OF CUTOFF WALL ON THE UPLIFT PRESSURE IN EARTH DAMS
}

\author{
M. Irandoost ${ }^{1}$, M. Reza Forghani ${ }^{2}$, S. N. Sayed Farizani ${ }^{3}$, O. Tayari $^{4}$ \\ ${ }^{1}$ Department of Water Engineering, Kerman branch, Islamic Azad University, Kerman, \\ ${ }^{2} \mathrm{MSc}$ of Water Structures, Department of water engineering, Kerman branch, Islamic Azad \\ University, Kerman, Iran \\ ${ }^{3} \mathrm{MSc}$ of Hydraulic Structures, Department of water engineering, Kerman branch, Islamic \\ Azad University, Kerman, Iran \\ ${ }^{4}$ Department of Civil Engineering, Kerman branch, Islamic Azad University, Kerman, Iran
}

Published online: 14 June 2016

\begin{abstract}
Since the construction of dam is significantly important both according to economic and safety aspects, it should be carefully assessed before construction. The use of available software is one of the evaluation and behavioral investigation methods. On this basis, we have investigated the effect of cutoff wall on the seepage flow, uplift pressure, etc, in the body of earth dam in Baft city through numerical modeling using the finite element method through GeoStudio software package. The results of this analysis indicate that the location and dimensions of cutoff wall significantly affects its performance on reducing the seepage flow. The soil type in terms of permeability is another important parameter and the results indicate that the one-time reduction of soil dam penetration reduces the seepage flow by $97 \%$.
\end{abstract}

Keywords: Earth dam, cutoff wall, soil permeability, GeoStudio software, uplift pressure.

Author Correspondence, e-mail: irandostmo@gmail.com

doi: http://dx.doi.org/10.4314/jfas.8vi2s.40 


\section{INTRODUCTION}

They play the significant roles in development of agriculture, development of rural and urban areas, drinking water supply, hydroelectric energy production, controlling and regulating the water flow in rivers, etc. Dams are significantly important according to the economic, social and political aspects. On the other hand, these strategic plans require the macroeconomic investment. In a world, where the nations' poor economic conditions are enhanced every day and the need for healthy drinking water supply is enhanced, the economy of plans is significantly important. Due to the high cost of building the dams, the economic issues and logical and optimal executive policies are very important along with adopting the dam maintenance policies (Khosravi et al., 2001).

The construction of cutoff walls with different concrete materials is the most common way to control the seepage under the foundation. Since the dams are rarely built on the completely non-permeable grounds, there is the groundwater flow in most of the cases and the seepage fully affects the proper performance of dam. The water pressure and output path of water from foundation can damage the permanent dam installations. The water seepage in the dam foundation and body has been the cause of numerous earth dam failures in most of the cases. The construction of cutoff coverage, draining, building the cutoff curtain and ultimately the in-situ concrete cutoff walls are among the water stop methods which have been popular during the last 20 years and has been gradually evolved (Jansen, 2002). The in-situ concreting is the conventional method for constructing the cutoff walls. The drilling is done with bentonite slurry and then pouring concrete is performed by conventional concrete or plastic clay concrete. The thickness of cutoff wall is about 0.6 meters and it is increased by 1-1.2 m for very deep walls. The uplift pressure is one of the most destructive forces which lead to the instability of dams and diversion weirs and it is resulted from the water flow in porous medium of foundations in dams and diversion weirs. This force reduces the shear strength between the dam and its foundation and leads to the tensile stress and thus consequently leads to the dam landslide and collapse. The exit gradient is also one of the most important criteria for designing the reliability to piping phenomenon. The construction of cutoff walls is one of the measures which are utilized for reducing the uplift pressure and exit gradient (Ghobadian and Khodaei, 2009). 
The cutoff walls, which are often impermeable or very low permeable, slow and reduce the water flow of infrastructure, and thus prevent the occurrence of water pressure in infrastructure. The reduced intensity of uplift pressure due to the construction of cutoff wall is called the pressure factor. Different ways are utilized to determine the flow characteristics of water infrastructures (seepage flow, uplift pressure and exit gradient) such as Belay and Ien's empirical methods, Khosla's mathematical method, flow network and numerical methods (Nili and Khazaei, 2005).

Herefore, it is essential to conduct the accurate determination and comprehensive investigation of presence on the dams along with the progress of technology for elimination of defects over time and structure oldness during the recent decades by structural engineers. The uplift pressure is the most important force due to the pressure of water volume inside the dam on its walls and the strategies are provided for controlling and preventing the serious damage of this force to the structure (Fell, et al. 2005). The cutoff wall is as a barrier to prevent the piping phenomenon, reduce the exit gradient as well as the seepage flow from the water infrastructures and this research investigates its effect on the uplift pressure.

The amount of exit gradient becomes lower than the basic exit gradient (without cutoff wall) by constructing a cutoff wall in any position. Furthermore, the maximum uplift pressure and minimum output hydraulic gradient are observed in a position wherein the cutoff wall is constructed downstream (Ghobadian and Khodaei, 2009). Due to the low permeability, high deformability, and sufficient strength to cope with the imposed stresses, the plastic concretes are the suitable materials for construction of such these walls in parts with weak foundation and high permeability. The materials constructing the plastic concrete including the bentonite, cement, aggregate and water have direct effects on its properties in terms of type and mixture ratio. Therefore, an appropriate mixture plan should be provided for providing the low permeability and suitable formability and strength of plastic concrete (Farajpour and Ghanbari, 2011). By building a cutoff wall at any position, the maximum uplift pressure is obtained in the case with constructed cutoff wall downstream. Furthermore, the uplift pressure is reduced by increasing the depth of cutoff curtain upstream compared to the middle cutoff curtain and downstream (Shiravand Amrouei et al, 2012). Providing the computer simulation of proposed 
model, Abolpour measured the uplift pressure on the water structures through different methods. He utilized Lin, Belay and Khoslay's methods for calculating the rates of uplift pressure and piping phenomenon and also Khoslay's method and flow network for measuring the amount of seepage (Abolpour, 1994).

The numerical solution through finite element method is utilized to reduce the problem in producing the mesh resulting from the drainage hole with small radius and compact distance, and eliminate the singularity in seepage points and mesh dependency as well as solving the problems of dam seepage with drainage systems (Chen et al, 2008). The finite difference method based on the coordinate transformation method is utilized for simulating the boundary fitted coordinates (BFC) and investigate the seepage under the dams through the application of Poisson equation (Jie, 2004). After examining and investigating the possible solutions and adopting the appropriate decision on New Zealand's Arapuni Dam, which has seepage due to the soil erosion and weak clay, the researchers suggested the cutoff wall for solving this problem (Amos et al, 2007).

This paper models and investigates the effects of cutoff wall on the uplift pressure in Baft dam.

\section{2- BAFT DAM}

Baft dam, which is located $160 \mathrm{~km}$ from south west of Kerman and $4 \mathrm{~km}$ from north east of Baft city. Baft dam has the pebble earth type with clay core. The technical characteristics of this dam are as follows: The riverbed elevation in dam location is $2295.25 \mathrm{~m}$ above the sea level, the drainage basin area is 259 square kilometers, the average height of drainage basin is also 2726 $\mathrm{m}$ above the sea level, the area of lake reservoir at normal level is $2.37 \mathrm{~m}^{2}$, the volume of lake reservoir at the normal level is 40 million cubic meters and the volume of lake reservoir at maximum level is 55 million cubic meters. This dam is constructed with the aims at providing 7 million cubic meters drinking water of Baft and Bezenjan Cities, the water for industry purposes equal to 9.2 million cubic meters, the agriculture water for water right lands, controlling the floods and seasonal runoff of Baft River as well as the regional development and employment through establishment of industries. 
In the case of full dewatering of dam, 7 million cubic meters of water can be applied as drinking water for Baft and Bezenjan cities, 9.2 million cubic meters for local industries (Baft steel plant) and 8.5 million cubic meters for 1300 hectares of water right lands.

The average rainfall of Baft region is about $240 \mathrm{~mm}$ per year and it has a constant flow and is mainly at the maximum level in winter and spring. In Baft basin, there is a hydrometric station located in Baft bridge region and it recorded the daily flow statistics from 1977 to 1983, but its statistics cannot represent the real output of river due to water diversion upstream at statistics stations. Furthermore, the data of Soltani River adjacent to Baft basin is utilized for statistical period of 1961 to 1994 in order to modify the recorded outcome values and also the duration of its statistics. The volume of receiving the water upstream at the station is determined based on diversion channel dimensions and the maximum capacity of the old refinery for drinking water and agricultural purposes downstream according to the experts' viewpoints, and finally the amounts of monthly outcome of Baft River are measured in different return periods. According to the results, the overall outcome of Baft River is estimated equal to 21.4 million cubic meters per year.

\section{3- MATERIALS AND METHODS}

The utilization of cutoff walls is one of the most important ways to reduce the rate of seepage flow under the earth dams. Using Seep/W software which measures the seepage through the finite element method, this research provides several models and studies the factors affecting the cutoff wall in reducing the seepage under the earth dams. Therefore, the cutoff wall is modeled with various characteristics in software and its uplift pressure is evaluated (Moradi and Parish, 2009). GeoStudio is the powerful software for finite element analysis and performs the static and dynamic analysis on the soil and it has the ability to model most of the structures according to the various spaces. The defined spaces for this software include the SEEP for three dimensional analysis of seepage in soil, analyzing the flow and seepage in soil, investigating the flow network in homogeneous and non-homogeneous dams, and determining the amount of seepage; SLOPE for analyzing the static and dynamic sustainability of slops with ability to reinforcing the slop; SIGMA for analyzing the stress-strain distribution in soil during the 
multistage levee; CTRAN for analyzing the distribution of pollutants inside the soil; and QUAKE for dynamic analysis of soil and investigating the areas with liquefaction potential.

\section{3-1- Modeling approach in software}

This software initially defines the soil properties and the type of different parts of dam through considering certain colors to those features, and then it determined the boundary conditions. Afterwards, the dam model is implemented in software and we perform zoning for it; then different colors with certain characteristics are assigned to those areas and also we define the location of applying the boundary conditions.

All regions should be gridded after completion of modeling stages. There are various elements for gridding and they should be applied depending on the type of analysis. The four or three-side elements or the combination of both can be applied in this regard. The selection of appropriate sizes for elements is an important point. Balancing the minimization of elements is significantly important for achieving the desired precision and analysis time in numerical issues. Despite the fact that minimizing the elements will lead to the precise responses, the high precision is not always the aim. For instance, the precision reaches from 1e-007 to 1e-008 in most of the analyses by precision software over time, while, this rate of precision is not required in most of the issues. Therefore, the size of element should be controlled according to the precision of responses in order to be properly implemented for other users' application of results or desired analysis. After examining the size and number of various elements and also the convergence of responses, the gridding is considered with 4291 nodes and 4276 elements for initial model as shown below.

Generally, the equation governing the groundwater flow in porous medium under two dimensional, non-confined, diverse, heterogeneous and unsteady conditions is according to the following equation based on Boussinesq non-linear equation:

$\frac{\partial}{\partial x}\left(K_{x} h \frac{\partial h}{\partial x}\right)+\frac{\partial}{\partial x}\left(K_{x} h \frac{\partial h}{\partial x}\right)=S_{y} \frac{\partial h}{\partial t}-R$

In which, $\mathrm{h}$ is the hydraulic load, $\mathrm{S}_{\mathrm{y}}$ is the specific yield, $\mathrm{K}_{\mathrm{x}}$ and $\mathrm{K}_{\mathrm{y}}$ are the hydraulic conduct components of porous medium at directions of $\mathrm{x}$ and $\mathrm{y}$; and $\mathrm{R}$ represents the amount of point feed and discharge. According to the differential equation with partial derivatives above, the 
unknown function in this equation is the amount of $\mathrm{h}$ which is a function of location $(\mathrm{x}, \mathrm{y})$ and time $(\mathrm{t})$.

Since there is a need for simplification of obtaining the response in any type of simulation, the conditions are assumed steady and without point feed and discharge. Given these hypotheses, the flow equation can be written according to the following equation which is the basis for seepage analysis in Seep code for saturation conditions of dam modeling.

$\frac{\partial}{\partial x}\left(K_{x} h \frac{\partial h}{\partial x}\right)+\frac{\partial}{\partial x}\left(K_{x} h \frac{\partial h}{\partial x}\right)=0$

The initial and boundary conditions are required for solving any differential equation and the selection of these boundary conditions is the most important step in preparation of conceptual model, so that selection of inappropriate boundary conditions may create an error in model and the difference between the model response and real system towards the incurred stresses.

In this software, the conceptual model of body and foundation is prepared according to the available technical data and scale. Afterwards, the values of dam materials' permeability based on the laboratory results of permeability to materials and change range of these coefficients are determined based on the field permeability; the results are presented in the following table.

Table 1. Permeability characteristics of materials in body and foundation of Baft dam

\begin{tabular}{cccc}
\hline No. & Type of Materials & $\begin{array}{c}\text { Horizontal } \\
\text { permeability } \\
\text { coefficient }\end{array}$ & $\begin{array}{c}\text { Vertical permeability } \\
\text { coefficient }\end{array}$ \\
\hline $\mathbf{1}$ & Core & $1 \mathrm{e}-008$ & $1 \mathrm{e}-008$ \\
$\mathbf{2}$ & Filter and drain & $1 \mathrm{e}-004$ & $1 \mathrm{e}-004$ \\
$\mathbf{3}$ & Cutoff wall & $1 \mathrm{e}-007$ & $1 \mathrm{e}-007$ \\
$\mathbf{4}$ & Foundation & $1 \mathrm{e}-005$ & $1 \mathrm{e}-005$ \\
\hline
\end{tabular}

\section{4- RESEARCH FINDINGS}

The complex linear and non-linear behavior of materials in earth dams multiplies the need for modeling the dam body for accurate control of dam behavior through appropriate behavior models. However, the complex behavioral models require the accurate geotechnical 
information which is very difficult and costly for determination. Therefore, the dam designing engineers are responsible for determining the proper monitoring of dam and these models can fulfill the monitoring requirements as well as being cost effective. Furthermore, the conducted studies on other dams such as the following cases confirm the importance of this issue.

\section{4-1- Analysis of modeling results}

Here, we have done the solution for several samples of cutoff wall in order to compare their results with each other. The following figure shows the results of modeling Baft dam with cutoff wall with a depth of $15 \mathrm{~m}$. These results include the contours of co-potential lines, and lines of flow and pressure on different parts of dam.
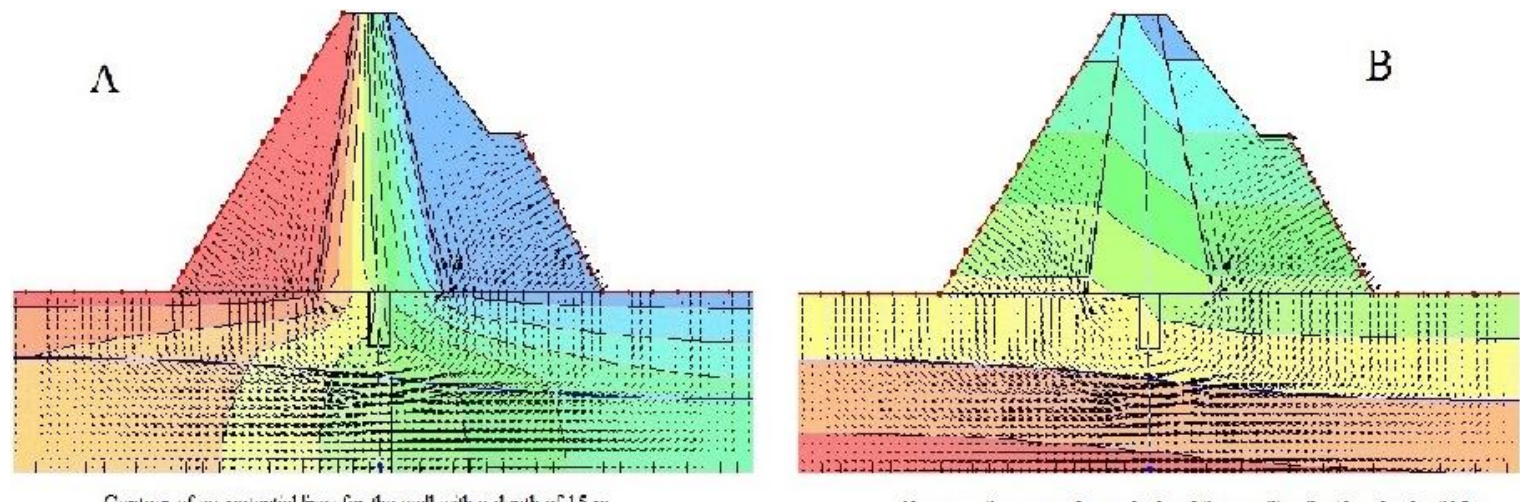

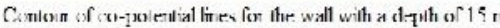

Contour of pressurc duc to the herd for cutofl wall with a dzpth of $15 \mathrm{~m}$

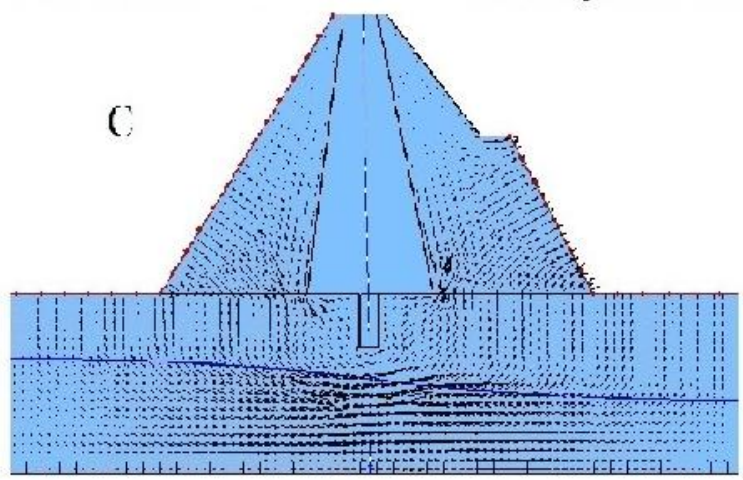

Lines of flowthrcughthe barricr laycrs

Fig.1. Schematic figures for contours of co-potential lines (A), head pressure (B) and flow through lines $(\mathrm{C})$ for different parts of dam at a depth of 15 meters

The flow through the middle section of dam is one of the most important results obtained from these contours. The amount of this number is equal to 3.196e-005 cubic meters per seconds for the model above and this indicates the dam seepage. In terms of flow, the Figure $1(\mathrm{C})$ indicates 
that the lines of seepage flow have higher concentration in the zone between the crust and core and also the crust and cutoff wall due to the difference between the permeability of these zones. Modeling the dam with the cutoff wall with a depth of $25 \mathrm{~m}$ is another model analyzed in this study and it is shown in the following figure. The size of element applied for this model is 1 meter between the cutoff wall of core and equal to 2.5 meters between the crust and foundation and the gridded responses are not changed by applying these sizes. Figure A2 shows the type of dam elements with cutoff wall with a length of 25 meters. It is noteworthy that all obtained results are equal to $2352.25 \mathrm{~m}$ for normal level.

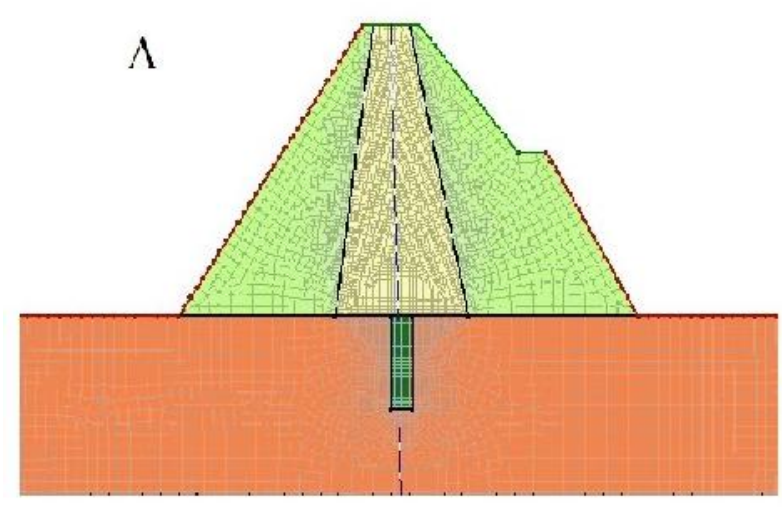

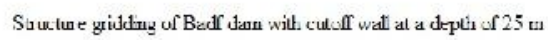

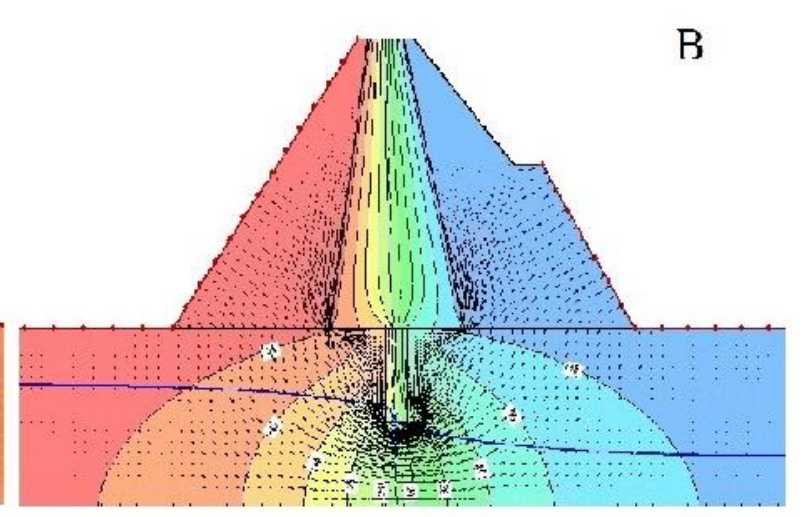

Contours of co-potential lines for the wall with depth of $25 \mathrm{~m}$

Fig.2. Schematic figure of dam gridding (A) and contour of co-potential lines (B) for different parts of dam at a depth of 25 meters

We have enhanced the soil permeability one-time for the wall at a depth of $25 \mathrm{~m}$ after the analyses in order to investigate its effect on the flow through the middle section of dam. The result of this analysis indicate that the flow of $1.48 \mathrm{e}-008$ is reduced, while the flow is obtained equal to $3.16 \mathrm{e}-006$ for the soil permeability of $1 \mathrm{e}-006$ and this indicates the reduction of $97 \%$ for permeability.

Afterwards, we have displaced the cutoff wall equal to $20 \mathrm{~m}$ for investigating the effect of cutoff wall location on the seepage flow. The model and grid of this displacement towards the dam reservoir upstream are shown in Figure 3. It is worth noting that these dimensions are 
utilized due to the received optimal results for meshing with mentioned dimensions for the model above.

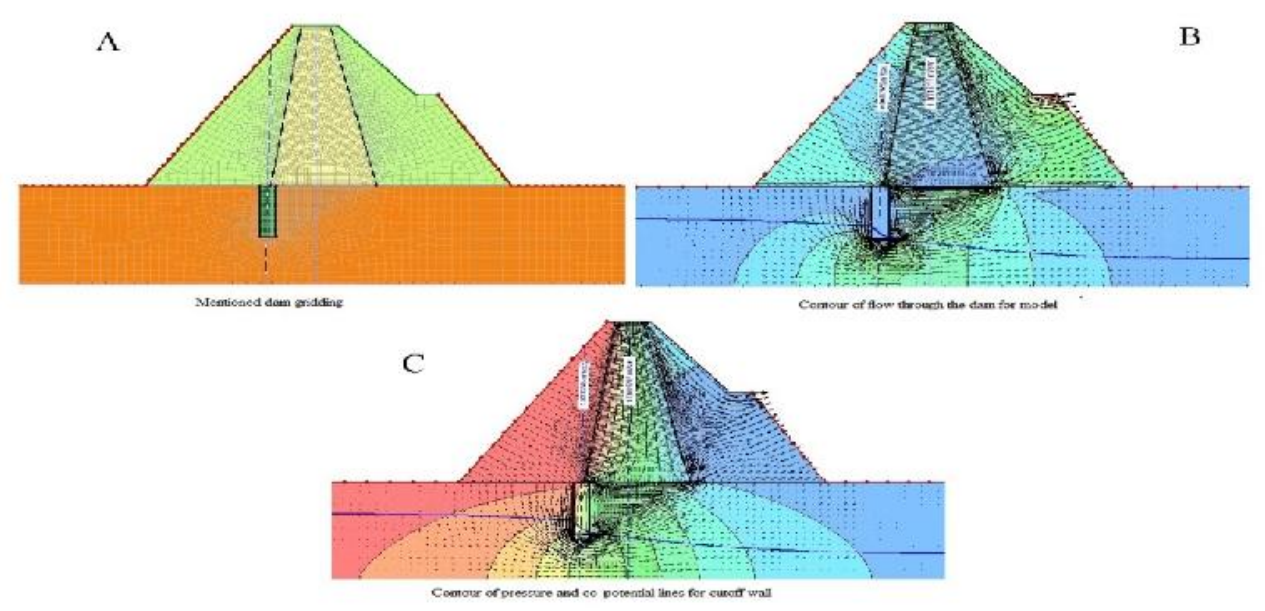

Fig.3. Schematic figure of gridding (A) contours of flow-through (B) and pressure and co-potential lines $(\mathrm{C})$ for different parts of dam

The results of this analysis indicate that the seepage through dam in sections is significantly increased as shown in Figure B3. In two sections shown in Figure C3, the values of seepage flow are explored and its value in the middle section is equal to $1.52 \mathrm{e}-004$ cubic meters per second. This indicates the 4-time increase than the dam with cutoff wall in the middle and also high permeability and indicates that it has a two-time increase than the dam with cutoff wall of $15 \mathrm{~m}$ in the middle and the soil properties with this model. It is worth noting that the permeability is in terms of meter per second and the seepage flow in cubic meter per second in the following diagram.

\section{4-2 investigating the effect of cutoff wall}

In general, the dam modeling without cutoff wall in this software and investigating its results indicate that the cutoff wall with the depth of 15 meters and the same soil properties decrease the flow in the middle section of dam for one time, and thus the uplift pressure is reduced as generally shown in figures above. 


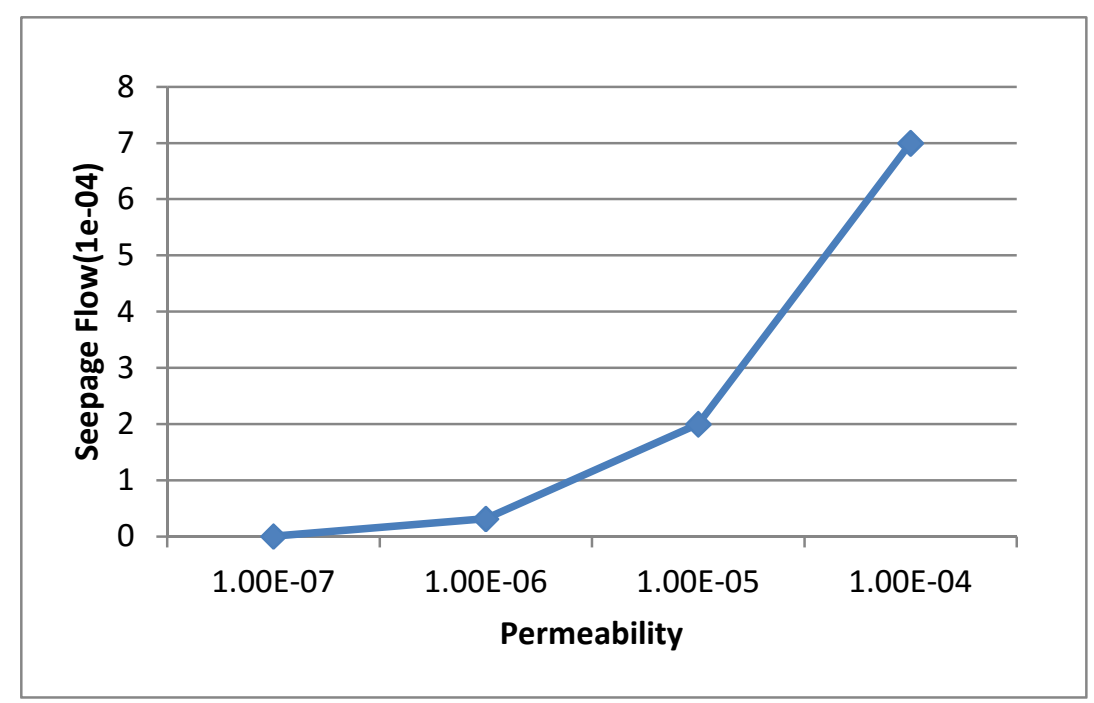

Fig.4. Effect of soil permeability on the rate of seepage flow from the middle section of dam

As shown in Figure 4, an increase in the soil permeability will lead to the increased seepage flow rate from the sections of dam. The effect of cutoff wall on the flow is presented based on two dimensionless parameters of these two. For flow, it is the relative flow and for cutoff wall, the relative length which is measured from dividing the length of cutoff wall by the zone with permeability as drawn in Figure 5. It is worth noting that the relative length of cutoff wall is in meters and the flow in meter per second.

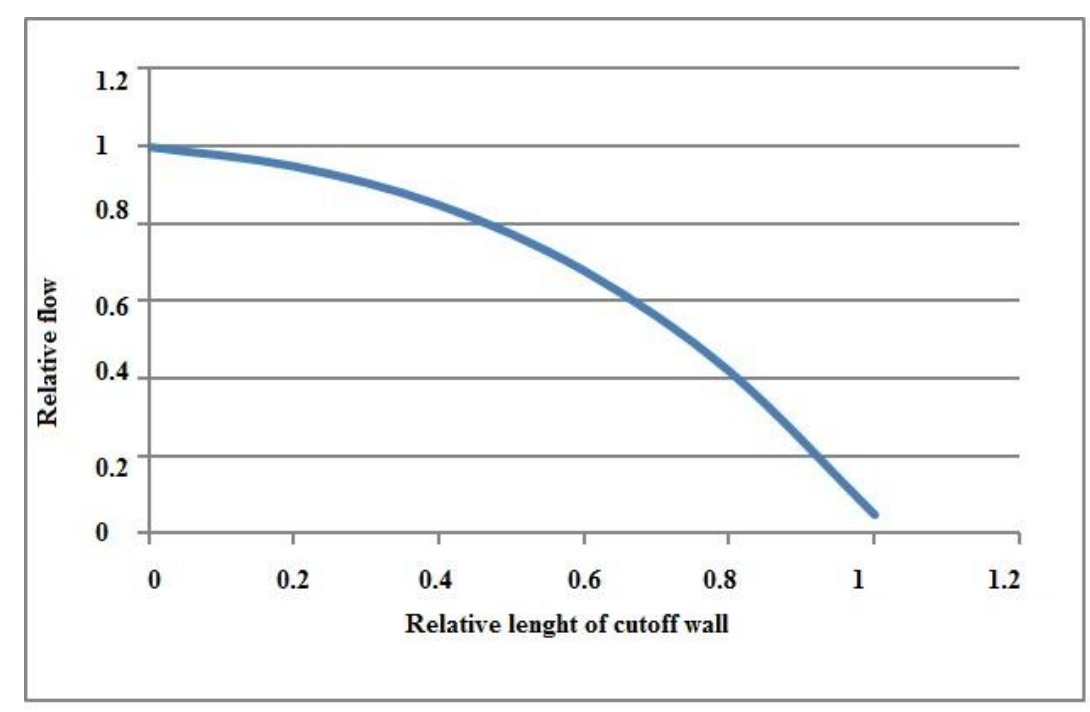

Fig.5. Diagram of relative flow-through rate based on the relative length of cutoff wall 
The results of this analysis indicate that the increased curtain length will lead to the enhanced uplift pressure; and as passing the curtain wall, the increased length will reduce the uplift pressure and this is true for middle cutoff wall and the water stop at the one side.

As shown in Figure 5, if the length of cutoff wall is considered approximately equal to the length of permeability, the effects of flow-through can be ignored because this value is close to zero according to the obtained results. According to this phenomenon, by increasing the length of cutoff wall, the flow line attached to dam and wall need stronger energy for passing it; it happens when the flow motion in porous medium is along with drop.

\section{4-3- Investigating the effect of cutoff wall location}

For evaluating the effect of cutoff wall on uplift pressure and also the depth of this wall, it is put in three zones and then we measure the water results for pressure. This wall is first located at the side and then in the middle and finally at the bottom; and its effects are observed.

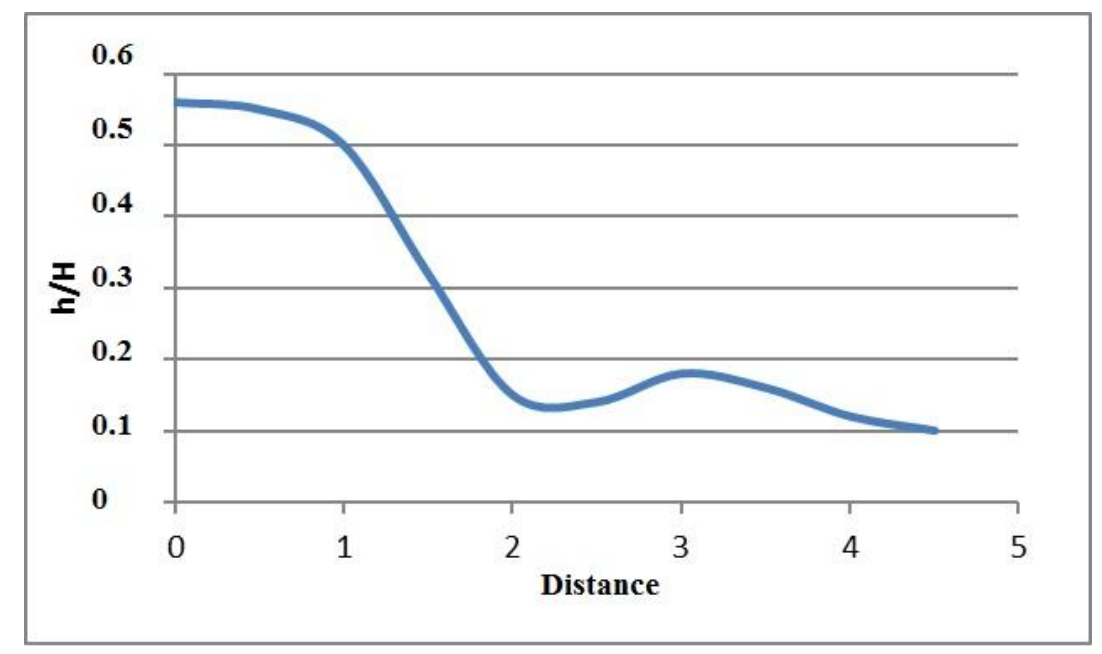

Fig.6. Reduced uplift pressure in the presence of cutoff wall at the sides

Therefore, the uplift pressure is enhanced by increasing the length of wall in the middle until the wall of cumulative energy for passing the curtain. After passing the wall, since the creeping path is increased, the enhanced length of curtain will lead to the higher drop in uplift pressure. It is noteworthy that the distance is in meter (Figure 7). 


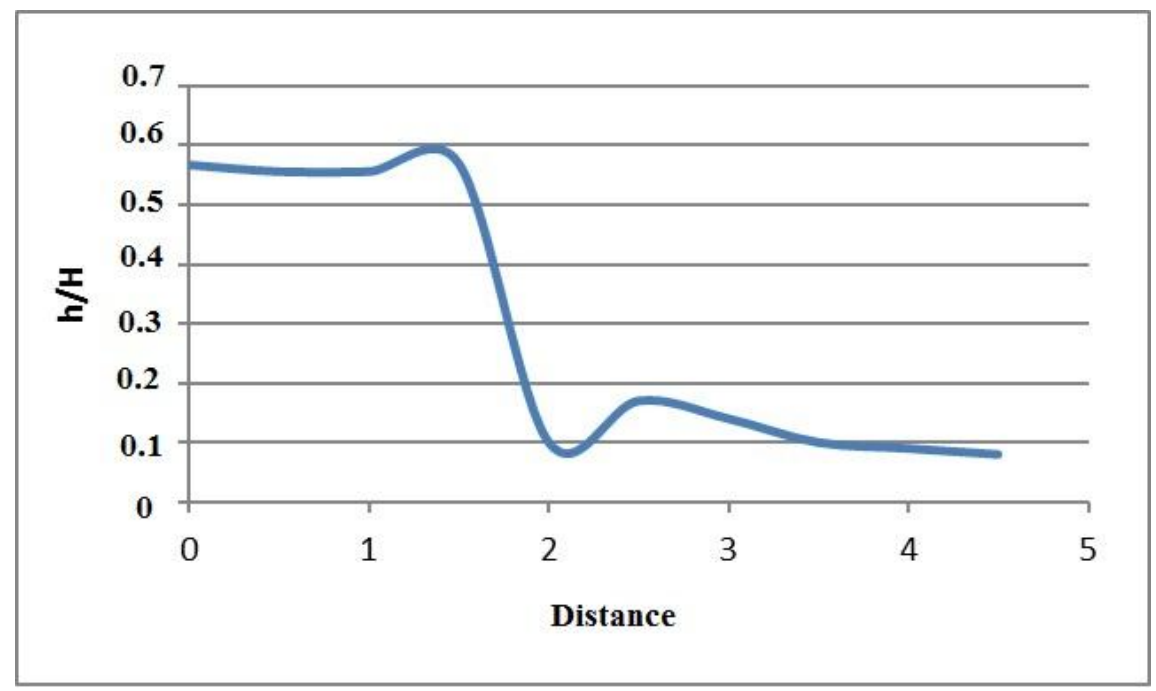

Fig.7. Reduced uplift pressure with cutoff wall in the middle

Figure 7 shows the effect of cutoff wall in the middle and it is shown that the uplift pressure is significantly decreased before the cutoff wall, but it is partly increased after passing the wall and it is due to passing with the pressure more than the smaller section.

Finally, the effect like the middle is seen by locating the cutoff wall at the bottom of dam as shown in Figure 8.

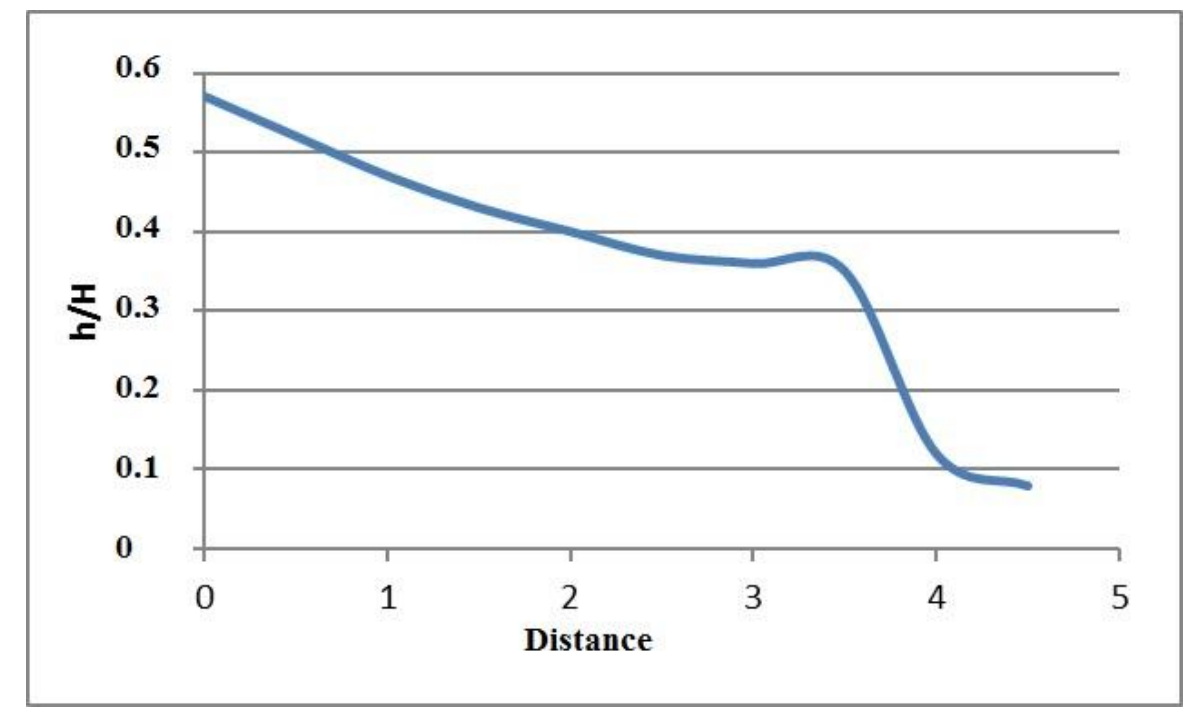

Fig.8. Reduced uplift pressure at the presence of cutoff wall at the bottom of dam

Given the three diagrams above, which show the effect of cutoff curtain on the reduced uplift pressure, it can be observed that the highest reduction of uplift pressure occurs by initial installation of cutoff wall, and the uplift pressure is more reduced by enhancing the depth of cutoff wall at the bottom. On this basis, the bottom of dam is the best place for curtain wall. 
According to what is seen here, the location of cutoff curtain at the bottom has higher importance and application than other models due to the reduced uplift pressure, thus it is put in the first priority according to the economic view due to the reduced potential to cause damage after the seepage and penetration of dam.

\section{5- CONCLUSION}

The study of cutoff wall behavior is an important issue which has been always taken into account by researchers. Since the investigation of wall behavior under various loads is not possible after implementation, it seems essential to theoretically identify the behavior of wall through the software analysis. Baft Dam is analyzed in this study for evaluating the rate of seepage and its uplift pressure. Several samples of dam with cutoff wall and various dimensions and soil properties are modeled in section Seep of GeoStudio software and their results are presented.

A summary of results is presented as follows.

- The cutoff wall generally reduces the flow-through and seepage of dam as well as reducing the uplift pressure.

- The cutoff wall with a depth of 15 meters and the soil properties of dam without improving its conditions suddenly reduce the flow in the middle section of dam.

- The reduced one-time permeability coefficients of soil in foundation of dam will lead to two-time reduction in permeability; in other words, if the permeability coefficient is halved, the permeability has the two-time reduction.

- The best performance of cutoff wall in reduction of seepage flow in the middle section of dam occurs when the cutoff wall is located at the center of core.

- The one-time reduction in soil permeability can dramatically reduce the flow-through by 97 percent. In other words, halving the soil permeability in software will reduce the flow up to 97 percent.

- We can clearly see the reduction in seepage flow due to the pressure of cutoff wall in contours. 
- The maximum reduction of uplift pressure initially occurs by installing the cutoff wall and the uplift pressure is even more reduced by enhancing the depth of cutoff wall at the bottom. Accordingly, the bottom of dam is best place for curtain wall in this study.

- The results of this research clearly indicate the reduction in uplift pressure before the cutoff wall and its increase after cutoff wall which are expected in flow and pressure contours.

- The 20-meter replacement of cutoff wall for keeping far distance of center from upstream indicates that the permeability is equal to $1.52 \mathrm{e}-004$ cubic meters per second and this rate shows the 4-time increase than the dam with cutoff wall in the middle.

\section{Suggestions for strategies of this study}

The results are obtained from a few studies and there is a need for more studies for their approval. Other issues occurred while investigating the impact of cutoff wall on seepage flow from the section of dam and uplift pressure, but they are not presented in this study for following the main subject of research; thus they are suggested for future studies:

1. Investigating the effect of water level upstream on the dam seepage in different seasons for Baft Dam;

2. Investigating the effect of water level downstream on variables such as the seepage, uplift pressure, etc for dam performance in different seasons;

3. Evaluating the effect of cutoff wall width on the flow-through in the middle section of dam;

4. Investigating other methods of water stop for Baft dam;

5. Investigating the effect of two cutoff walls; once near upstream and then downstream on the performance of dam in terms of seepage flow and so on.

\section{REFERENCES}

[1] Abolpour, B. (1994). Methods of estimating the seepage, uplift pressure and piping phenomenon in hydraulic structures by providing a computer simulation, Master's thesis on Irrigation and Drainage, Shiraz University. 
[2] Amos, P. D. Bruce, D. A. Lucchi, M. Newson, T. Wharmby, N. 2007. Design and Construction of Seepage Cut-off Walls Under a Concrete Dam in New Zealand With A Full Reservoir, American Society of Dam Safety Officials (ASDSO) Conference Austin Texas, USA.

[3] Chen, Y. Chuangbing, Z.And Hong., Z. (2008), A numerical solution to seepage problems with complex drainage systems, Computers and Geo-techniques 35, pp 383-393.

[4] Farajpour, Z.; and Ghanbari, D., (2011), Investigation of plastic concrete cutoff walls of Silveh dam; The First International Conference on non-permeable concretes in drinking water storage reservoirs.

[5] Fell, R. MacGregor., P. Stapledon. D.And Bell., G. 2005. Geotechnical Engineering of Dams.

[6] Ghobadian, R.; and Khodaei, K., (2009), Effects of cutoff wall and drain on uplift pressure and exit gradient in water infrastructures by numerical solution of general equation for water flow in soil through finite volume method, water and soil (agricultural Sciences and industries), 23 (4), 148-160.

[7] Jansen R. B. 1988. Advanced Dam Engineering for Design, Construction, and Rehabilitation, Springer.

[8] Jie, Y. (2004), Seepage analysis based on boundary fitted coordinate transformation method computer and Geo-techniques, Computers and Geo-techniques 31, pp. 279-283. [9] Khosravi, F.; Sadeghi, H.; Vakilpour, J., (2002), construction of gallery below the core of earth dam with an approach to Karkheh dam, The Third International Conference on Iran Geotechnical Engineering and Soil Mechanics.

[10] Moradi, A.; and Parish, Y., (2009), Evaluation of plastic concrete cutoff wall in controlling the seepage from the foundation of earth dams; The first International Conference on Concrete Technology.

[11] Nili, M.; and Khazaei, J., (2005), Analysis of plastic concrete cutoff wall using ANSYS software (finite element method); Second International Conference on Concrete and Development. 
[12] Nusier, O.K., Alawneh, A.S., Malkawi, A.H., 2002. Remedial measures to control seepage problems in the Kafrein dam, Jordan. Bulletin of Engineering Geology and the Environments, vol. 16. Springer, pp. 145-152.

[13] Shiravand Amrouei, R.; Vashaghani Farahani, M.; Mousavi Jahromi, H.; and Ahadian, M., (2012), Investigating the effect of depth and location of cutoff wall on the uplift pressure in diversion dams using seep/w software; The third national seminar on geotechnical issues in irrigation and drainage networks.

\section{How to cite this article:}

Irandoost M, Reza Forghani M, Sayed Farizani S. N, Tayari O. Flow simulation and investigating the effects of cutoff wall on the uplift pressure in earth dams. J. Fundam. Appl. Sci., 2016, 8(2S), 715-731. 